\title{
O Perfil dos Usuários de Caixa-Automáticos em Agências Bancárias na Cidade de Curitiba
}

\author{
Péricles José Pires \\ Renato Zancan Marchetti
}

\begin{abstract}
RESUMO
Este artigo apresenta os resultados de uma pesquisa realizada para identificar o perfil dos usuários dos caixas-automáticos em agências bancárias de Curitiba (PR). Na classificação dos altos, médios e baixos usuários dos caixas-automáticos, utilizou-se a análise de correspondentes múltiplos (MCA). Constatou-se, na análise dos resultados, que os altos usuários pertencem às classes sociais mais elevadas, principalmente do sexo masculino, e situam-se na faixa etária de 36 a 45 anos. Seus hábitos de leitura referem-se a jornais de outros estados, revistas esportivas e de atualidades. Estão familiarizados com as novas tecnologias de comunicação, sendo usuários de serviços e equipamentos, como: televisão por assinatura, Internet, telefonia celular, microcomputadores e aparelhos de fax. Foram, também, analisadas as operações realizadas nos caixas-automáticos em comparação com o atendimento pessoal, a influência exercida pelo banco na decisão de uso destes serviços, os aspectos relacionados às tarifas cobradas, a qualidade da orientação recebida dos funcionários, assim como a percepção dos clientes quanto ao ambiente onde os equipamentos estão localizados.
\end{abstract}

Palavras-chaves: perfil de usuários de caixas-automáticos/altos, médios e baixos usuários/análise de correspondentes múltiplos (ACM)

\begin{abstract}
The main purpose of this research is to identify the profile of Automated Teller Machine users, from the degree of utilization of the principal available operations in this equipment. The research focused on ATMs installed in the self-service areas of Banco do Estado do Paraná (Banestado), Banco do Brasil, Bradesco and Bamerindus, located in Curitiba - Brazil. The research also consisted of 296 questionnaires which were applied in the self-service environment of the above mentioned banks, from September 19 to October 4, 1996, during business hours. To build such profile, a reference key was utilized in order to rate high, medium and low users, a tool that proved to be consonant in the generation of scores. Also analyzed were the issues concerning ATM operations in comparison with services performed by a clerk, the influence from the bank and from other people in the decision to use an ATM, taxes and fees, advertising, the instructions for use given by clerks, and the client's perception of the environment in which the equipment is located. While forging this profile, that includes demographic and psychographic variables, it was possible to identify that the high users belong to higher social classes, they are 26 to 45 years of age, they read national newspapers; sports, automotive and current affairs magazines; they watch cable TV and own or use personal computers, fax machines, cellular phones, and they access the Internet. The results show that ATMs represent a tangible benefit to clients, for they are faster, easy to use and they carry the client's required privacy and reliability, being essential at the choice of a bank by the client. The perception regarding the influence from advertising refers to that which is broadcast on television and in the environment of the ATMs. Although clients have indicated their own initiative towards the use of the equipment, the necessity of an instructing clerk to help with the operations was explicit, chiefly with less common operations, such as ATM transferals and deposits.
\end{abstract}

Key words: the profile of automated teller machine user/high, medium and low users/multiple correspondence analysis (MCA) 


\section{INTRODUÇÃO}

Mudanças significativas têm ocorrido no atendimento aos clientes que utilizam os serviços oferecidos pelos bancos, no Brasil e no mundo. Novos e emergentes canais de acesso, com conotação de banco eletrônico, como o home e office banking, a Internet, o smart cards, o banco por telefone e as centrais de atendimento afetam as estratégias de relacionamento com os clientes. Os bancos têm procurado direcionar os clientes para os ambientes de auto-serviço nas agências, disponibilizando a quase totalidade de seus produtos e serviços, anteriormente ofertados exclusivamente através do atendimento pessoal ou no ambiente da agência, de forma eletrônica.

O sucesso da passagem da forma tradicional de atendimento para o auto-serviço é condicionado pela aceitação desta inovação pelos clientes. A compreensão do processo de aprendizagem, das atitudes e do comportamento do cliente em relação a estas novas modalidades de serviços, quer seja uma nova opção para consulta num terminal de auto-atendimento, ou o acesso a transações mais complexas, como o pagamento de uma fatura que utilize a captura eletrônica via código de barras, deve ser uma preocupação constante dos bancos interessados em atender às expectativas de seus clientes.

Para Zeithaml e Gilly (1987), embora as inovações tecnológicas possam trazer vantagens aos consumidores, muitos deles são resistentes a sua adoção, especialmente quando a inovação representa a perda do contato pessoal ou de informação. Para Kaynak (1993), a efetividade dos planos e estratégias mercadológicas, para um banco ou qualquer empresa, baseia-se no conhecimento sistemático do comportamento de compra do produto ou do serviço pelos clientes. Sapp e Smith (1984) concluem que o entendimento da natureza e das características dos consumidores de serviços bancários é valioso instrumento para a aproximação com o mercado.

O objetivo deste artigo é o de apresentar o perfil dos usuários dos caixas-automáticos, a partir do grau de utilização (alto, médio e baixo) dos produtos e serviços oferecidos no auto-atendimento dos bancos, tais como: saques em dinheiro, consultas, depósitos, transferências entre contas, aplicações financeiras, solicitações de talões de cheque e pagamentos de contas. O trabalho apresenta, na primeira parte, uma breve revisão da literatura sobre a importância da automação na estratégia de marketing dos bancos. A segunda parte enfoca os procedimentos metodológicos utilizados na realização da pesquisa com os clientes dos bancos envolvidos na análise. 


\section{A Automação Bancária na Estratégia de Marketing do Banco}

A tecnologia bancária brasileira teve início na década de 60 , visando atender à necessidade de expansão geográfica e à ampliação de seus serviços. A formação de conglomerados, englobando diversos tipos de atividades bancárias, exigiu investimentos em mainframes e a criação dos centros de processamento de dados (CPD).

Com a expansão da base de clientes e de contas, a partir dos anos 70 , cresceu a necessidade de investimentos em tecnologia em duas frentes, conforme exemplifica Gonçalves (1994): na "automação bancária e no processamento centralizado do banco". Neste estágio, a automação se limitou às atividades de frente, realizadas nas agências e postos de serviço, como autenticação de documentos, consulta a saldos, registro de transações e as atividades administrativas da agência, como fechamento do caixa, atualização de saldos, entre outros.

Para Chorafas (1987), os anos 60 foram marcados pelo cartão de crédito; os anos 70, pela introdução dos caixas-automáticos e terminais de ponto de venda; os anos 80, pela interatividade do videotexto, do homebanking e do gerenciamento do fluxo de caixa. Presenciamos, nos anos 90, a evolução desses recursos, através da difusão de novos conceitos, como o banco virtual, o cartão inteligente (smartcard), as novas funções para o auto-serviço e outras novidades no campo da informatização e automação para o segmento bancário.

A estratégia dos bancos tem sido oferecer aos clientes maior gama de serviços nos caixas-automáticos. Estas mudanças têm refletido alterações no layout das agências, ampliando a área do auto-atendimento, através de equipamentos que são capazes de atender à demanda por serviços bancários, como retiradas em dinheiro, extratos das contas correntes, aplicações e investimentos, depósitos, pagamento de contas, talões de cheques emergenciais e outros. Os bancos obtêm vantagens com a redução de custos, pois as transações que não envolvem pessoal diretamente, tendem a ficar menos onerosas. Para Faust (1990), o uso dos caixasautomáticos oferece também maior conveniência ao cliente, tanto no que diz respeito aos horários de funcionamento, quanto à facilidade de acesso aos serviços e maior rapidez na realização de tarefas rotineiras.

Para Steiner e Teixeira (1990), os bancos estão adotando a tecnologia como elemento chave na reestruturação do seu processo de gestão. Os avanços da tecnologia tornam possível a substituição de papéis, a agilização dos fluxos de trabalho, a realização de transações ou arquivamento e a manipulação de dados através da eletrônica. Indicam Albertin e Moura (1995), que a automação bancária é o redesenho dos processos de negócio dos bancos e implica realinhamento estratégico. A utilização de recursos computacionais, aliada a esta estratégia emer- 
gente, cria uma vantagem competitiva a partir da diferenciação, constituindo-se num alicerce, pois permite distribuir produtos e serviços de forma inovadora, agregar novas opções aos clientes e desenvolver relacionamentos de longo prazo com eles.

Conclui-se que a estratégia dos bancos, com relação à automação e aos caixasautomáticos instalados dentro da agência, nas ante-salas de auto-atendimento e nos demais pontos remotos, tem como conotação principal o atendimento à massa de clientes, através de serviços padronizados, com maior rapidez e conveniência. Como resultado, obtém-se a otimização do ponto de venda pela ampliação dos horários de atendimento, inclusive 24 horas, além da possibilidade de realização de negócios, através de transações específicas na aferição de tarifas.

Nos anos 50, devido à influência do modelo americano e europeu, disseminouse no Brasil a técnica do auto-serviço. Iniciou-se com as mercearias, passando pelas lojas de departamentos, postos de gasolina, shopping centers e outros, consolidando este tipo de atendimento como importante ferramenta para o varejo. Para Groonroos (1990), o sucesso do self-service depende do grau de envolvimento dos usuários e dos benefícios por eles percebidos, devendo os mesmos serem recompensados e motivados por tomarem parte do processo.

Relacionados a essa concepção, os caixas-automáticos baseiam-se, essencialmente, na venda automática de produtos e serviços bancários. Para Kotler (1995), essas máquinas oferecem aos consumidores as vantagens da venda 24 horas, e da ausência da manipulação por terceiros. Considerados máquinas de venda altamente especializadas, os caixas-automáticos propiciam aos usuários uma série de vantagens, já mencionadas, sendo uma delas de fundamental importância: a conveniência de tempo, lugar e acesso. A aceitação do consumidor em relação à tecnologia vem-se expandindo (McCoy, Frieder e Hedges, 1993), tornando o uso da ATM elemento essencial na maneira de as pessoas conduzirem seus negócios financeiros.

\section{Objetivos e Metodologia da Pesquisa}

O objetivo principal desta pesquisa foi identificar o perfil do usuário dos caixas-automáticos, a partir do grau de utilização dos diversos serviços bancários oferecidos através destes. Kotler (1995) e Weinstein (1995) mencionam, entre as principais variáveis de segmentação, a taxa de uso do produto ou serviço, classificando o cliente em não usuário, pequeno, médio ou grande usuário. O entendimento acerca das categorias de uso pode auxiliar no desenvolvimento de ações estratégicas para o aumento do consumo entre os pequenos e médios usuários de 
um determinado produto, assim como na oferta de serviços formatados aos diversos segmentos.

Para Markin (1971), quando se enfoca o mercado a partir das características comportamentais dos consumidores, é possível extrair-se melhor entendimento sobre as expectativas, desejos e necessidades das diversas categorias de clientes. Engel, Blackell e Miniard (1995) indicam a especial ênfase que deve ser dada aos altos usuários, sendo desejável atraí-los e retê-los como consumidores, em comparação com os moderados ou baixos usuários.

O perfil será composto a partir da avaliação do grau de utilização dos caixasautomáticos, no que diz respeito às operações de saques, extratos, consultas, depósitos, pagamento de contas, transferências, aplicações e solicitações de talões de cheque.

A condução do trabalho apoiou-se na identificação das variáveis relacionadas ao uso e ao comportamento dos usuários dos caixas-automáticos, procurando-se, no confronto dessas variáveis, entender quais as mais representativas na composição do perfil. Para Gianesi e Corrêa (1994) os fatores comportamentais ou psicográficos cortam transversalmente os segmentos definidos demograficamente, identificando grupos de clientes com padrões de compra de produtos e serviços em comum.

$\mathrm{Na}$ construção das categorias foram focalizados os serviços identificados através de pesquisa preparatória, realizada junto a uma dezena de especialistas de diversos bancos brasileiros. Esses serviços deveriam estar disponíveis aos clientes nas agências, seja através do guichê de caixa ou balcão de atendimento, ou de máquinas específicas de auto-serviço, que são os cash-dispensers (CD) ou as automatic teller machines (ATM), ambas consideradas na pesquisa como caixas-automáticos.

O trabalho teve como escopo pesquisar e estudar as diversas classes de usuários de serviços bancários automatizados e o seu comportamento quanto ao acesso aos equipamentos de auto-atendimento. Com relação à interação equipamentocliente, podem ser citadas algumas características, como o grau de utilização concentrado em determinadas camadas sociais, a resistência ao uso pelos clientes mais idosos e outros. Desta forma, o núcleo da problemática da pesquisa reside na identificação do perfil dos usuários, que estão sendo afetados com a introdução de novas tecnologias e novas formas de atendimento bancário.

O auto-atendimento provoca perda do contato pessoal dos clientes com os funcionários do banco, desenvolvendo novo tipo de interação, pois se coloca entre os agentes (clientes e funcionários) uma máquina muito parecida com um computador. A evolução tecnológica está mudando os paradigmas do mundo atual, fazen- 
do-se uma constante em quase todas as atividades. Para a indústria bancária não seria diferente: a combinação da tecnologia com outros fatores, está disseminando novas estratégias de atuação num mercado altamente especializado, competitivo e regulamentado. As mudanças no ambiente das agências bancárias, com a criação de espaços para o auto-atendimento, são claro prenúncio de que a evolução tende a transferir as operações rotineiras para as máquinas, a fim de aumentar a produtividade do ponto-de-venda, a conveniência e o conforto nos serviços oferecidos aos clientes.

O escopo do trabalho reside, então, na investigação e na comprovação dos fatores apontados como relevantes no comportamento das pessoas perante as inovações ou novas tecnologias, tais como: classe social, faixa etária, escolaridade, além de prospectar novos indicadores consistentes neste relacionamento, como a posse e o uso de aparelhos eletrônicos que permitem o acesso aos serviços bancários automatizados e informatizados, como os microcomputadores, aparelhos de fax, acesso à rede Internet e outros.

Os resultados do estudo auxiliarão na compreensão dos fatores supracitados e na formulação de estratégias de relacionamento com os clientes, como, por exemplo, a adequação das ações ao grupo de usuários que mais necessita de suporte para a utilização dos equipamentos.

As etapas da pesquisa realizada estão a seguir descritas.

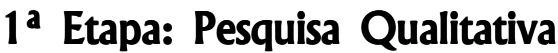

A etapa qualitativa foi subdividida em duas fases. A primeira fase concentrouse nas entrevistas com especialistas em auto-atendimento para o segmento bancário; a segunda fase, nas entrevistas junto a clientes usuários dos caixas automáticos. O objetivo principal desta etapa foi extrair subsídios para a construção das escalas de medida utilizadas na fase quantitativa.

A pesquisa junto aos especialistas foi conduzida através de entrevistas semi-diretivas (Marchetti, 1995), em duas ações programadas: a primeira voltada para um posicionamento inicial sobre o assunto, e a segunda para a validação, considerando a opinião consolidada de todos os especialistas consultados. O objetivo da pesquisa realizada com os especialistas foi o de obter informações sobre o auto-atendimento e os principais serviços ofertados através deste, assim como identificar o perfil percebido do alto, médio e baixo usuário dos caixas-automáticos.

$\mathrm{Na}$ segunda fase foram realizadas entrevistas em profundidade com clientes 
usuários dos caixas-automáticos residentes em Curitiba (PR), utilizando-se um roteiro de entrevista que cobrisse os objetivos do estudo. Abordaram-se dois clientes de cada banco objeto da pesquisa (Banestado, Banco do Brasil, Bradesco e Bamerindus); agregando-se o Banco Itaú e o Unibanco, perfez-se um total de doze entrevistas. O objetivo destas entrevistas foi o de identificar os principais benefícios percebidos pelos clientes na utilização do auto-atendimento, levantar as principais razões para o uso e as dificuldades na utilização.

\section{2a etapa: Pesquisa Quantitativa}

A segunda etapa consistiu numa pesquisa quantitativa, não probabilística, com clientes usuários dos caixas-automáticos. As entrevistas foram realizadas no ambiente de auto-atendimento dos bancos Banestado, Banco do Brasil, Bradesco e Bamerindus, todos localizados na região central da cidade de Curitiba (PR). Foram entrevistados 296 usuários, distribuídos em cotas estabelecidas a partir dos horários de funcionamento deste serviço.

\section{Análise dos Resultados}

Os dados coletados na fase quantitativa foram submetidos a tratamento estatístico, utilizando-se a análise de correspondentes múltiplos (Bagozzi, 1995). A análise de correspondentes múltiplos (MCA) é uma técnica de descrição de dados qualitativos, particularmente bem adaptada ao tratamento de dados de pesquisa, em que as questões são relacionadas a respostas múltiplas. Formalmente se trata de simples aplicação da análise de correspondentes a uma tabela disjuntiva com $\mathrm{m} 1+\mathrm{m} 2+\ldots+\mathrm{mp}$ modalidades. Este método possui certas propriedades que o ligam a outros métodos estatísticos e lhe emprestam um caráter particular, que $o$ fazem equivalente à análise em componentes principais para variáveis qualitativas (Saporta, 1990; Weller e Romney, 1990; Morineau, Lebart e Pirou, 1995).

Utilizando-se a análise de correspondentes múltiplos - procedimento HOMALS (SPSS, 1993) obteve-se o escore dos respondentes na primeira dimensão da análise, DIM 1. Este escore é relativo à intensidade de uso de cada operação disponível no auto-atendimento, categorizando-se os usuários, conforme sua intensidade de utilização, desde o de maior utilização (maior escore) até o de menor utilização (menor escore). 
A Tabela 1, apresenta os escores obtidos através da análise de correspondentes múltiplos (MCA) para os cinqüenta primeiros classificados.

Tabela 1: Escore DIM 1 dos 50 Maiores Usuários Obtidos pela MCA

\begin{tabular}{|c|c|c|c|c|c|c|c|c|c|}
\hline ORDEM & ESCORE & ORDEM & ESCORE & ORDEM & ESCORE & ORDEM & ESCORE & ORDEM & ESCORE \\
\hline $1^{\circ}$ & 3,36 & $11^{\circ}$ & 2,10 & $21^{\circ}$ & 1,86 & $31^{\circ}$ & 1,43 & $41^{\circ}$ & 1,13 \\
\hline $2^{\circ}$ & 3,36 & $12^{\circ}$ & 2,08 & $22^{\circ}$ & 1,80 & $32^{\circ}$ & 1,40 & $42^{\circ}$ & 1,09 \\
\hline $3^{\circ}$ & 2,79 & $13^{\circ}$ & 2,08 & $23^{\circ}$ & 1,79 & $33^{\circ}$ & 1,36 & $43^{\circ}$ & 1,09 \\
\hline $4^{\circ}$ & 2,79 & $14^{\circ}$ & 2,01 & $24^{\circ}$ & 1,77 & $34^{\circ}$ & 1,36 & $44^{\circ}$ & 1,09 \\
\hline $5^{\circ}$ & 2,73 & $15^{\circ}$ & 2,01 & $25^{\circ}$ & 1,73 & $35^{\circ}$ & 1,36 & $45^{\circ}$ & 1,09 \\
\hline $6^{\circ}$ & 2,62 & $16^{\circ}$ & 2,01 & $26^{\circ}$ & 1,73 & $36^{\circ}$ & 1,36 & $46^{\circ}$ & 1,00 \\
\hline $7^{\circ}$ & 2,57 & $17^{\circ}$ & 2,01 & $27^{\circ}$ & 1,63 & $37^{\circ}$ & 1,32 & $47^{\circ}$ & 0.97 \\
\hline $8^{\circ}$ & 2,57 & $18^{\circ}$ & 2,01 & $28^{\circ}$ & 1,51 & $38^{\circ}$ & 1,30 & $48^{\circ}$ & 0.97 \\
\hline $9^{\circ}$ & 2,36 & $19^{\circ}$ & 1,95 & $29^{\circ}$ & 1,45 & $39^{\circ}$ & 1,26 & $49^{\circ}$ & 0,97 \\
\hline $10^{\circ}$ & 2,10 & $20^{\circ}$ & 1,93 & $30^{\circ}$ & 1,43 & $40^{\circ}$ & 1,17 & $50^{\circ}$ & 0,97 \\
\hline
\end{tabular}

O escore foi extraído da intensidade de utilização para as principais operações nos caixas-automáticos (saques, consultas a saldos e extratos, transferências, depósitos e obtenção de talões de cheques). Os maiores escores referem-se àqueles usuários que utilizam mais intensivamente todos os serviços, desde as transações mais comuns, como saques e consultas, até a utilização de operações mais raras, como as transferências entre contas e depósitos.

Uma importante conclusão que se extrai da análise é que não basta apenas considerar-se o total de acessos às operações mais comuns nos caixas-automáticos (por exemplo, obter extratos ou saques de dinheiro), para afirmar que determinado indivíduo é alto ou baixo usuário. É preciso analisar a variedade das operações realizadas pelo cliente, assim como a intensidade com que ele utiliza cada um dos serviços ofertados. A vantagem da utilização da análise de correspondentes múltiplos na determinação do escore de intensidade de utilização dos caixas-automáticos é a de permitir a obtenção de indicador quantitativo que representa, de forma efetiva, a combinação de intensidade e variedade dos serviços utilizados. O escore DIM 1 será utilizado como variável que se explica nas análises subseqüentes. 
Com o intuito de validar o escore obtido pela análise de correspondentes múltiplos identificou-se, junto aos especialistas do setor, na fase qualitativa desta pesquisa, um critério empírico, visando a classificar o cliente na categoria de alto, médio e baixo usuário dos serviços dos caixas-automáticos. Este critério considerou um número mínimo e um número máximo para a intensidade de utilização de cada tipo de operação. Na determinação da importância de cada operação, utilizou-se uma ponderação definida pelo consenso dos especialistas. Os resultados obtidos através da utilização do método, baseados no julgamento dos especialistas, foram correlacionados com o escore DIM 1, calculado com a utilização da análise de correspondentes múltiplos - correlação de Pearson (Siegel, 1975), encontrando-se um coeficiente de $\mathbf{0 , 8 6 1 3}$ com o valor $\mathbf{p}=\mathbf{0 , 0 0 0}$, o que sinaliza na direção de uma boa validade convergente entre os dois indicadores.

A identificação do perfil dos usuários dos caixas-automáticos em agências bancárias de Curitiba (PR) foi efetuada a partir do escore obtido pela MCA, DIM 1, que foi considerado como variável que se explica nas análises desenvolvidas. A relação entre as variáveis nominais do tipo classe social, sexo, escolaridade, hábitos de utilização dos veículos de comunicação como assistir à televisão, ler revistas e jornais e o escore DIM 1, foi efetuada através da análise da variância (Siegel, 1975; Stevenson, 1981; Churchill Jr, 1983; Mattar, 1994). O procedimento selecionado foi ANOVA ONEWAY (Conover, 1971; Tukey, 1991) do pacote estatístico SPSS. O teste de significância empregado foi o TUKEY (Siegel, 1975; Gomes, 1982), recomendado para a comparação entre células com efetivos desiguais.

O perfil do usuário dos caixas-automáticos também foi construído através das análises de associação, entre o escore DIM 1 e outras variáveis mensuradas no estudo, como a atitude dos usuários em relação: ao atendimento no caixa-automático comparativamente ao atendimento pessoal, à cobrança de tarifas, às orientações recebidas, ao ambiente físico, e aos equipamentos utilizados. O grau de associação entre estas variáveis, de natureza intervalar, e o escore DIM 1 foi obtido através do coeficiente de correlação conhecido como "r de Pearson" (Conover, 1971; Siegel, 1975; Stevenson, 1981), disponível no pacote estatístico SPSS. A seguir, estão apresentados os resultados obtidos no estudo. 


\section{Operaçōes de Auto-Atendimento Versus Atendimento no Guichê de Caixa: Comparaçōes dos Resultados entre as Diversas Categorias de Usuários}

As operações realizadas no caixa-automático, comparadas com o atendimento pessoal no guichê do caixa, conforme demonstrado na Tabela 2, não estão relacionadas com a intensidade de utilização do caixa-automático, medido pelo escore DIM 1.

\section{Tabela 2: Operacões de Auto-Atendimento Versus Atendimento no Guichê de Caixa - Comparações dos Resultados Obtidos entre as Diversas Categorias de Usuários}

\begin{tabular}{|c|c|}
\hline AFIRMAÇÕES & $\begin{array}{c}\text { INTENSIDADE DE } \\
\text { UTILIZAÇÃO } \\
\text { (escore DIM 1) } \\
\text { CORRELAÇÃO }\end{array}$ \\
\hline 1. As operações no $\mathrm{CA}^{*}$ são mais rápidas do que no Caixa humano. & $-0,397-\mathrm{NS}$ \\
\hline 2. As operações no CA* são mais fáceis do que no Caixa humano. & 0,1018 - NS \\
\hline $\begin{array}{l}\text { 3. As operações no CA* são mais confiáveis, sem erros ou } \\
\text { problemas, do que no Caixa humano }\end{array}$ & $0,0706-\mathrm{NS}$ \\
\hline $\begin{array}{l}\text { 4. As operações no CA* são mais seguras, em função da } \\
\text { privacidade e do sigilo do que no Caixa humano. }\end{array}$ & $0,1543-\mathrm{NS}$ \\
\hline $\begin{array}{l}\text { 5. No CA* existe uma maior disponibilidade de horários e } \\
\text { pontos de atendimento. }\end{array}$ & $-0,0597-\mathrm{NS}$ \\
\hline 6. No $\mathrm{CA}^{*}$ tenho que enfrentar filas. & $0,0669-\mathrm{NS}$ \\
\hline \multicolumn{2}{|l|}{$\begin{array}{l}\text { 7. No CA* o atendimento é padronizado, não dependendo de } \\
\text { pessoas para a realização das operações. }\end{array}$} \\
\hline $\begin{array}{l}\text { 8. No CA* posso realizar a maioria das transações bancárias, sem } \\
\text { necessitar entrar no interior da agência. }\end{array}$ & 0,0605 - NS \\
\hline 9. Com a disponibilização do $\mathrm{CA}^{*} \mathrm{o}$ atendimento do banco melhora. & 0,1265 - NS \\
\hline 10. Com o uso do $\mathrm{CA}^{*}$ diminui o número de cheques emitidos. & $-0,0007-\mathrm{NS}$ \\
\hline $\begin{array}{l}\text { 11. O oferecimento do CA* é importante na minha escolha de } \\
\text { um banco. }\end{array}$ & $0,1547-\mathrm{NS}$ \\
\hline $\begin{array}{l}\text { 12. Os CAs* podem substituir o atendimento humano nas } \\
\text { operações bancárias. }\end{array}$ & $0,1322-\mathrm{NS}$ \\
\hline
\end{tabular}


Pode-se constatar pela análise dos coeficientes de correlação na Tabela 2, questões 1 a 12, que não foram observadas associações significativas entre a intensidade de utilização dos serviços (escore DIM 1) e os benefícios percebidos no caixa-automático, comparativamente ao atendimento pessoal no guichê do caixa. Estes resultados indicam que os benefícios do auto-atendimento (rapidez, facilidade, confiabilidade, segurança, disponibilidade de horários, qualidade do atendimento, etc) são percebidos e valorizados igualmente, quando comparados ao atendimento pessoal, tanto pelos altos como pelos médios e pequenos usuários dos caixas-automáticos.

\section{Opiniōes Relativas à Cobrança de Tarifas: Comparaçōes dos Re- sultados entre as Diversas Categorias de Usuários}

O grau de intensidade de utilização dos caixas-automáticos não está associado à cobrança de tarifas nas operações destes serviços, conforme se pode observar na Tabela 3.

Tabela 3: Correlação entre o Grau de Utilização e as Afirmações Relativas à Cobrança de Tarifas

\begin{tabular}{|l|l|}
\hline \multicolumn{1}{|c|}{ AFIRMAÇÕES } & $\begin{array}{c}\text { INTENSIDADE DE UTILIZAÇÃo } \\
\text { (escore DIM 1) } \\
\text { CORRELAÇÃ̂O }\end{array}$ \\
\hline $\begin{array}{l}\text { 1. A cobrança de tarifas influencia na decisão de } \\
\text { utilizar o CA*. }\end{array}$ & $-0,0570$ - NS \\
\hline $\begin{array}{l}\text { 2. Os valores cobrados nas operações no CA* não } \\
\text { são compatíveis com os serviços oferecidos. }\end{array}$ & $-0,0429$ - NS \\
\hline $\begin{array}{l}\text { 3. Com relação à cobrança de tarifas, confiro e } \\
\text { acompanho os valores cobrados. }\end{array}$ & $-0,0950$-NS \\
\hline $\begin{array}{l}\text { 4. No caso da cobrança para o uso do guichê de } \\
\text { caixa, usaria com maior intensidade o CA. }\end{array}$ & $-0,0821$-NS \\
\hline
\end{tabular}

Estes resultados indicam que a estratégia na cobrança de tarifas pela utilização dos caixa-automáticos, em princípio, não deveria ser direcionada para determinado grupo de usuários, em função de sua intensidade de utilização dos serviços, parecendo ser inócua a estratégia de "quanto mais usar, menos se cobrará", para 
o caso dos caixas-automáticos. É interessante observar que a cobrança pela utilização dos serviços no guichê de caixa poderia canalizar número maior de usuários para os serviços do auto-atendimento.

\section{Opiniōes Relativas às Orientações Recebidas: Comparações dos Resultados entre as Diversas Categorias de Usuários}

A Tabela 4 apresenta a análise da correlação entre as afirmativas relacionadas às orientações recebidas dos funcionários para o uso das operações de auto-atendimento e o grau de utilização dos serviços dos usuários. Observa-se, novamente, que não existe relacionamento entre o escore obtido pela frequência de utilização das operações e as opiniões das diversas categorias de usuários, relativas aos serviços de orientação.

Tabela 4: Correlação entre o Grau de Utilização dos Caixas-Automáticos e as Orientações Recebidas

\begin{tabular}{|l|l|}
\hline \multicolumn{1}{|c|}{ AFIRMAÇÕES } & $\begin{array}{c}\text { INTENSIDADE DE UTILIZAÇÃO } \\
\text { (escore DIM 1) } \\
\text { CORRELAÇÃO }\end{array}$ \\
\hline $\begin{array}{l}\text { 1. Quando tenho alguma dificuldade em operar o } \\
\text { CA*, peço orientação a um funcionário. }\end{array}$ & $0,0094-\mathrm{NS}$ \\
\hline $\begin{array}{l}\text { 2. Quando tenho alguma dificuldade em operar o } \\
\text { CA*, desisto da operação . }\end{array}$ & $-0,0449$ - NS \\
\hline $\begin{array}{l}\text { 3. As orientações dadas para as operações nos CA* } \\
\text { não são corretas e precisas. }\end{array}$ & 0,0735 -NS \\
\hline $\begin{array}{l}\text { 4. Os funcionários estão sempre fornecendo } \\
\text { orientações sobre novos produtos e serviços } \\
\text { disponibilizados nos CA*. }\end{array}$ & $-0,0873$-NS \\
\hline $\begin{array}{l}\text { 5. O ambiente de auto-atendimento deve ter um } \\
\text { funcionário exclusivo para orientação. }\end{array}$ & $-0,0591$-NS \\
\hline $\begin{array}{l}\text { 6. As telas dos equipamentos são orientativas, sem } \\
\text { dificuldades para utilização das operações. }\end{array}$ & $-0,0412$-NS \\
\hline $\begin{array}{l}\text { 7. Imagem, mensagens e o som dos equipamentos, } \\
\text { ajudam no acesso e na utilização das operações } \\
\text { disponibilizadas nas telas dos CA* }\end{array}$ & 0,0379 -NS \\
\hline
\end{tabular}


Esses resultados indicam que os clientes, independentemente da sua freqüência de utilização, podem necessitar de instruções indistintamente, ou seja, as possibilidades de um alto usuário recorrer a um funcionário para a orientação são as mesmas de um baixo ou médio usuário. É importante ressaltar que o caixa-automático, na perspectiva de grande parte dos clientes dos bancos, é percebido como nova tecnologia de acesso aos serviços bancários, e não como novo produto. A ruptura causada pela modificação na forma tradicional de atendimento levará certo tempo para ser absorvida pelos usuários; os bancos deverão investir fortemente, durante a transição, nos instrumentos que poderão acelerar o processo de aprendizagem do cliente, tais como a orientação personalizada e a comunicação.

\section{Opiniōes Relativas ao Ambiente Físico e aos Equipamentos: Comparaçōes dos Resultados entre as Diversas Categorias de Üsuários}

Na Tabela 5, encontra-se a correlação entre o grau de utilização e as afirmativas que abrangem o ambiente físico e os equipamentos. Os resultados indicam não existir associação entre a intensidade de utilização dos serviços (altos, médios e baixos usuários) e os fatores relacionados ao ambiente físico dos caixasautomáticos, como a segurança percebida e os equipamentos utilizados.

Tabela 5: Correlação entre o Grau de Utilização e as Afirmações Relativas ao Ambiente Físico: Segurança Percebida e Equipamentos

\begin{tabular}{|l|l|}
\hline \multicolumn{1}{|c|}{ AFIRMAÇÕES } & $\begin{array}{c}\text { INTENSIDADE DE UTILIZAÇÃo } \\
\text { (escore DIM 1) } \\
\text { CORRELAÇÃO }\end{array}$ \\
\hline $\begin{array}{l}\text { 1. O ambiente onde estão os CA* deveria estar } \\
\text { sempre limpo, com boa aparência dos equipamentos } \\
\text { e mobiliário. }\end{array}$ & $-0,0175-\mathrm{NS}$ \\
\hline $\begin{array}{l}\text { 2. O ambiente onde estão os CA* deveria sempre } \\
\text { estar bem iluminado. }\end{array}$ & $-0,0122$ - NS \\
\hline $\begin{array}{l}\text { 3. O ambiente de auto-atendimento deveria possuir } \\
\text { um vigilante. }\end{array}$ & 0,0758 -NS \\
\hline $\begin{array}{l}\text { 4. Os equipamentos deveriam estar separados uns dos } \\
\text { outros através de biombos. }\end{array}$ & 0,0560 -NS \\
\hline $\begin{array}{l}\text { 5. Deveria haver uma quantidade maior de } \\
\text { equipamentos. }\end{array}$ & 0,0414 -NS \\
\hline
\end{tabular}


Esses resultados recomendam uma ação efetiva no que diz respeito aos fatores relacionados à atmosfera do local do auto-atendimento. A preocupação com a segurança e o conforto do cliente, a limpeza e a iluminação devem, de certa forma, compensar a falta do contato pessoal, que é sempre mais agradável numa relação de atendimento. Um fator importante na política de competição entre os bancos é a manutenção do clima de cordialidade entre a instituição e seus clientes, independentemente do seu grau de utilização dos serviços.

\section{Perfil Sociodemográfico dos Usuários dos Caixas-Automáticos}

A Tabela 6 apresenta os resultados da análise de variância, conduzida para identificar o perfil sociodemográfico dos altos, médios e baixos usuários dos caixas-automáticos, respectivamente.

Tabela 6: Perfil Sociodemográfico dos Usuários dos Caixas-Automáticos

\begin{tabular}{|ll|c|l|}
\hline VARIÁVEIS DEMOGRÁFICAS & F & $\begin{array}{c}\text { INTENSIDADDE DE UTILIZAÇÃo } \\
\text { (escore DIM 1) - Teste de Tukey }\end{array}$ \\
\hline 1. Classe Social & $\mathrm{N}=295$ & 10,0337 & $\begin{array}{l}\text { Identificadas diferenças significativas entre a } \\
\text { classe social A e as classes B,C e D. }\end{array}$ \\
\hline 2. Escolaridade & $\mathrm{N}=296$ & 2,7035 & N.S \\
\hline 3. Faixa Etária. & $\mathrm{N}=296$ & 3,0739 & $\begin{array}{l}\text { Identificadas diferenças significativas entre os } \\
\text { respondentes de 36 a 45 anos com os de 56 a } \\
65 \text { anos. }\end{array}$ \\
\hline 4. Sexo & $\mathrm{N}=296$ & 6,5525 & $\begin{array}{l}\text { Identificada diferenças significativas entre os } \\
\text { usuários de sexo masculino e feminino. }\end{array}$ \\
\hline
\end{tabular}

Pode-se observar na Tabela 6 que os usuários que mais utilizam os serviços do auto-atendimento são aqueles que pertencem às classes sociais mais elevadas (CRITÉRIO ABIPEME). Os indivíduos que se inserem nas classes sociais superiores recebem, por sua condição social, maior carga de informações, através do convívio familiar e profissional. Além disso, podem-se considerar outros aspectos ligados a essa variável de classificação, como maior preocupação com a administração do tempo, que os predispõem a uma utilização mais intensiva de serviços que proporcionem facilidade de acesso e rapidez no atendimento. Os resultados indicam, também, que os grandes usuários dos serviços dos caixas-automáticos estão 
situados na faixa etária de 36 a 45 anos e pertencem ao sexo masculino. Os clientes na faixa etária de 56 a 65 anos estão entre aqueles que apresentam a menor freqüência de utilização dos caixas-automáticos.

Os usuários mais jovens, principalmente nas grandes cidades, têm maior familiaridade com as inovações tecnológicas, como a automação e a informatização. Isto é verdadeiro para quase todas as suas atividades diárias, trabalho, educação e atividades de lazer, convivendo desde a infância com máquinas específicas, mesmo aquelas que se prestam ao auto-serviço, como dispensadoras de refrigerantes, comestíveis e outras. O usuário com idade mais avançada tem maior tendência a resistir às inovações, principalmente aquelas de caráter tecnológico. Essas conclusões podem ser evidenciadas em outros estudos, que se dedicaram especificamente às questões relacionadas ao comportamento inovador dos consumidores (Dubois e Marchetti, 1993).

\section{Hábitos de Mídia: Comparaçōes dos Resultados entre as Diversas Categorias de Usuários}

A Tabela 7, a seguir, apresenta os resultados relacionados aos hábitos de mídia dos entrevistados, como assistir à televisão, à televisão por assinatura, leitura de revistas e jornais.

Os resultados da ANOVA (Tabela 7) indicam que os entrevistados com maior intensidade de utilização dos serviços dos caixas-automáticos assistem com maior freqüência à televisão por assinatura, são leitores de revistas sobre atualidades (Veja, Isto É, etc), automóveis, motos e esportivas, e estão mais expostos ao jornalismo de cobertura nacional. A análise desses resultados indica que os altos usuários dos caixas-automáticos são pessoas bem informadas e expostas, com maior intensidade, às informações sobre inovações tecnológicas. Possuem, também, elevado grau de cosmopolitismo no que diz respeito aos seus hábitos de mídia. Essas informações podem contribuir, de forma efetiva, na elaboração do programa de mídia mais adequado ao atingimento desse público-alvo. 


\section{Tabela 7: Hábitos de Mídia e a Intensidade de Utilização dos Caixas-Automáticos}

\begin{tabular}{|c|c|c|}
\hline HÁBITOS DE MÍDIA & $\mathbf{F}$ & $\begin{array}{l}\text { INTENSIDADE DE UTILIZAÇÃO } \\
\text { (escore DIM 1) - Teste de Tukey }\end{array}$ \\
\hline 1. Assistir à televisão. & 1,1546 & N.S \\
\hline $\begin{array}{l}\text { 2. Assistir à televisão por } \\
\text { assinatura. }\end{array}$ & 6,1088 & $\begin{array}{l}\text { Identificadas diferenças entre os respondentes que } \\
\text { assistem a até duas horas de TV por assinatura e } \\
\text { aqueles que nunca assistem. }\end{array}$ \\
\hline 3. Ler revistas da atualidade. & 3,2057 & $\begin{array}{l}\text { Constatadas diferenças entre aqueles que lêem to- } \\
\text { dos os números publicados e aqueles que lêem me- } \\
\text { nos de um número a cada dois publicados. }\end{array}$ \\
\hline 4. Ler revistas masculinas. & 1,8267 & N.S \\
\hline $\begin{array}{l}\text { 5. Ler revistas de estilo de } \\
\text { vida }\end{array}$ & 0,8412 & N.S \\
\hline 6. Ler revistas populares. & 0,3517 & N.S \\
\hline 7. Ler revistas econômicas. & 2,1932 & N.S \\
\hline 8. Ler revistas de informática. & 3,4072 & N.S \\
\hline 9. Ler revistas de decoração. & 0,4807 & N.S \\
\hline $\begin{array}{l}\text { 10. Ler revistas de automóvel, } \\
\text { moto e esportes. }\end{array}$ & 5,3710 & $\begin{array}{l}\text { Indicadas diferenças entre os leitores de todas os } \\
\text { números e aqueles que não lêem a revista. }\end{array}$ \\
\hline 11. Ler jornais locais. & 2,1962 & N.S \\
\hline $\begin{array}{l}\text { 12. Ler jornais de outros es- } \\
\text { tados. }\end{array}$ & 4,6665 & $\begin{array}{l}\text { Indicadas diferenças entre os leitores de todos os } \\
\text { dias e os de uma vez por semana com os que nunca } \\
\text { lêem. }\end{array}$ \\
\hline 13. Ler jornais econômicos. & 0,1653 & N.S \\
\hline
\end{tabular}

\section{Posse e Uso de Equipamentos de Alta Tecnologia: Comparaçōes dos Resultados entre as Diversas Categorias de Usuários}

A Tabela 8 apresenta os resultados relacionados à posse e uso de serviços e equipamentos de alta tecnologia de comunicação. 
Tabela 8: Posse e Uso de Equipamentos de Alta Tecnologia e a Intensidade de Utilização dos Caixas-Automáticos

\begin{tabular}{|l|c|c|}
\hline POSSE E USO DE EQUIPAMENTOS & F & $\begin{array}{c}\text { INTENSIDADE DE UTILIZAÇÃo } \\
\text { (escore DIM 1) }\end{array}$ \\
\hline 1. Possui microcomputador. & 9,2460 & $\mathrm{p}=0,0026$ \\
\hline 2. Utiliza microcomputador. & 7,9982 & $\mathrm{p}=0,0050$ \\
\hline $\begin{array}{l}\text { 3. Possui computador instalado para realiza- } \\
\text { ção de operações de casa. }\end{array}$ & 0,1026 & $\mathrm{~N} . \mathrm{S}$ \\
\hline $\begin{array}{l}\text { 4. Usa computador instalado para realização } \\
\text { de operaçôs de casa. }\end{array}$ & 1,1779 & $\mathrm{~N} . \mathrm{S}$ \\
\hline 5. Possui acesso à Internet. & 11,3820 & $\mathrm{p}=0,0008$ \\
\hline 6. Utiliza o acesso à Internet. & 10,3567 & $\mathrm{p}=0,0014$ \\
\hline 7. Possui fax. & 34,34 & $\mathrm{p}=0,0000$ \\
\hline 8. Utiliza fax. & 20,4825 & $\mathrm{p}=0,0000$ \\
\hline 9. Possui telefone celular. & 18,1672 & $\mathrm{p}=0,0000$ \\
\hline 10. Utiliza telefone celular. & 16,0677 & $\mathrm{p}=0,0001$ \\
\hline
\end{tabular}

Os resultados indicam que os altos usuários são pessoas familiarizadas com a tecnologia de informática e comunicação, fazendo uso rotineiro de equipamentos e serviços dessa natureza, como o microcomputador, o fax e o telefone celular. Estão conectados na Internet, apesar de ainda não utilizarem intensivamente os serviços de home-banking. Esses resultados podem contribuir não somente nos aspectos relacionados à comunicação de marketing, mas também na compreensão do processo de adoção dessa tecnologia de auto-atendimento. Os usuários com maior intensidade de utilização são aqueles que foram mais expostos a equipamentos e serviços relacionados à informática. Para usuários com menor grau de exposição a esse tipo de inovação é interessante um forte apoio do atendimento pessoal, visando a quebrar as barreiras impostas pela rejeição e ganhar a confiança e a adesão dos clientes, de modo geral, para os novos serviços.

\section{Conclusóes}

A partir das análises efetuadas é possível desenhar o perfil dos maiores usuários dos caixas-automáticos em agências bancárias em Curitiba (PR). 


\section{Perfil dos Maiores Usuários dos Caixas-Automáticos}

\begin{tabular}{|l|}
\hline PERFIL SOCIODEMOGRÁFICO \\
\hline - Pessoas de classe social mais elevada; \\
- Faixa etária entre 36 e 45 anos; \\
- Pertencem principalmente ao sexo masculino. \\
\hline HÁBITOS DE MÍDIA \\
\hline - Usuários dos serviços de televisão por assinatura; \\
- Lêem revistas de atualidade e revistas esportivas e de automóveis; \\
- Lêem com maior freqüência jornais de outros estados. \\
\hline POSSE DE BENS \\
\hline - Possuem e utilizam microcomputadores, fax, telefone celular, tendo acesso à Internet. \\
\hline
\end{tabular}

Esses resultados confirmam estudos anteriores (Costa Filho, 1996), que identificaram que os altos usuários dos serviços dos caixas-automáticos pertencem a um grupo mais jovem de clientes e são encontrados mais facilmente nas classes sociais A e B.

As principais contribuições desse estudo podem ser categorizadas da seguinte forma: (1) sob a perspectiva metodológica, a utilização da análise de correspondentes múltiplos demonstrou ser uma técnica adequada para a geração de escores que representem a intensidade de utilização dos serviços, sendo de grande utilidade nos estudos de segmentação; (2) sob a perspectiva da gestão de marketing, os resultados contribuem, de forma efetiva, para a compreensão da natureza do processo de adoção de novas tecnologias de atendimento ao cliente, disponibilizando informações importantes para o desenvolvimento de estratégias de marketing de relacionamento do banco.

\section{ReferênCias Bibliográficas}

ALBERTIN, A. L.;

MOURA, R. M. de.

Administração de informática e seus fatores críticos de sucesso no setor bancário privado nacional. Revista de Administração de Empresas, v.35, n.5, p.22-29, set./out. 1995. 
BAGOZZI, R.

Advanced methods of marketing research. Oxford : Blackwell, 1995.

CHORAFAS, D. N.

Strategic planning for eletronic banking : from human resources to product development and information systems. London : Butterworths, 1987.

CHURCHILL Jr, G. A.

Marketing research : methodological foundatons. Fort Worth : Dryden Press, 1983.

CONOVER, W. J.

Practical nonparametric statistics. New York : John Wiley \& Sons, 1971.

COSTA FILHO, B. A. da.

Automação bancária : uma análise sob a ótica do cliente. São Paulo, 1996. Dissertação (Mestrado em Administração) Faculdade de Economia, Administração e Contabilidade, Universidade de São Paulo.

DUBOIS, B.;

MARCHETTI, R.

Le comportement innovateur des foyers dans l'achat des produits durables : le cas das biens electroniques au Brésil. Recherche et Applications en Marketing, v.8, n.1, 1993.

FAUST, W. H.

The branch as a retail outlet. The Bankers Magazine, v.173, n.1, p.30-35, Jan./Feb. 1990.

GIANESI, I. G. N.;

CORREA, H. L.

Administração estratégica de serviços : operações para a satisfação do cliente. São Paulo : Atlas, 1994.
GOMES, F. P.

Curso de estatística experimental. Piracicaba : Nobel, 1982.

GONÇALVES, J. E. L.

Os impactos das novas tecnologias nas empresas prestadoras de serviços. Revista de Administração de Empresas, v.34, n.1, p.63-81, jan./fev. 1994.

GROONROOS, C.

Service management and marketing : managing the moments of truth in service competition. Massachusetts : Lexington Book, 1990.

KAYNAK, E.

Consumer perception of commercial banks in a newly industrialized country. Journal of Professional Services Marketing, v.9, n.2, p.139-157, 1993.

KOTLER, P.

Administração de marketing : análise, planejamento, implementação e controle. 4.ed. São Paulo : Atlas, 1995.

MARCHETTI, R.

Diversidade e tendências das pesquisas qualitativas de marketing. Caderno de Ciências Socias Aplicadas, Curitiba, 1995.

MARKIN, R. J.

Retailing management. London: MacMillan, 1971.

MATTAR, F. N.

Pesquisa de marketing. São Paulo : Atlas, 1995. 
McCOY J. B.;

FRIEDER, L. A.;

HEDGES, R. B.

Bottomline banking. Chicago : Irwin, 1993.

MORINEAU, A.;

LEBART, L.;

PIRON, M.

Statistique exploratoire multidimensionnelle. Paris : DUNOD, 1995.

SAPP, R. W.;

SMITH, R. W.

Strategic management for bankers. Oxford : Planning Executives Institute, 1984.

SAPORTA, G.

Probabilités, analyse des données et statistique. Paris : Technip, 1990.

SIEGEL, S.

Estatística não-paramétrica. São Paulo : McGraw-Hill, 1975.

SPSS.

SPSS for windows : base system user's guide \& professional statistics. Ontário : SPSS, 1993.

STEINER, T. D.;

TEIXEIRA, D. B.

Technology in banking : creating value anddestroying profits. Homewood: Business One Irwin, 1990.

STEVENSON, W. J.

Estatística aplicada à administração. São Paulo : Harper \& Row, 1981.

TUKEY, J. W.

Fundamentals of exploratory analysis of variance. New York: Wiley-Interscience, 1991.
WEINSTEIN, A.

Segmentação de mercado. São Paulo: Atlas, 1995.

WELLER, S. C.;

ROMNEY, A. K.

Metric scaling : correspondence analysis. London : Sage, 1990.

ZEITHAML, V. A.;

GILLY, M. C.

Characteristics affecting the acceptance of retailing technologies : a comparison of elderly and nonelderly consumers. Journal of Retailing, v.63, n.1, p.48- 68, Spring 1987. 